\title{
Retraction of: Long Noncoding RNA SOX2OT Accelerates the Carcinogenesis of Wilms' Tumor Through ceRNA Through miR-363/FOXP4 Axis, by Ma L, Sun X, Kuai W, Hu J, Yuan Y, Feng W, and Lu X. DNA Cell Biol [Epub ahead of print]; DOI: 10.1089/dna.2018.4420, 2018.
}

\begin{abstract}
It has come to the Journal's attention that the cell lines used in the study titled Long Noncoding RNA SOX2OT Accelerates the Carcinogenesis of Wilms' Tumor Through ceRNA Through miR-363/FOXP4 Axis by Ma L, Sun X, Kuai W, Hu J, Yuan Y, Feng W, and Lu X, DOI: 10.1089.dna.2018.4420, are not of Wilms' tumor origin, but rather are Ewing (SK-NEP-1) (Memorial Sloan Kettering) and Rhabdoid (G-401) (ATCC). These data were provided by unimpeachable authorities. Memorial Sloan Kettering Cancer Center (among the premier institutions for cancer research in the world) and ATCC (the most highly respected repository for cell lines and reagents) disagree with the authors' assumptions about the specificity of the origin of the cell lines.
\end{abstract}

While many other publications can be found that assert the cell lines are of Wilms' tumor origin, this is not supported by the benchmark organizations.

For that reason, the experimental conclusions drawn in this peer-reviewed paper cannot be attributed to provide insights into Wilms' tumors, but rather to two cell lines of other pedigree. Therefore, DNA and Cell Biology is officially retracting this paper.

\section{References}

ATCC. www.lgcstandards-atcc.org/Products/All/CRL-1441.aspx?geo_country=it

Memorial Sloan Kettering Cancer Cancer. https://www.mskcc.org/research-advantage/support/technology/tangiblematerial/sk-nep-1-human-ewing-sarcoma-cell-line 